\title{
Relationship between photospheric currents and coronal magnetic helicity for force-free bipolar fields
}

\author{
S. Régnier \\ School of Mathematics and Statistics, University of St Andrews, St Andrews, Fife, KY16 9SS, UK \\ e-mail: stephane@mcs.st-andrews . ac .uk
}

Received 11 December 2008 / Accepted 13 March 2009

\section{ABSTRACT}

\begin{abstract}
Aims. The origin and evolution of the magnetic helicity in the solar corona are not well understood. For instance, the magnetic helicity of an active region is often about $10^{42} \mathrm{Mx}^{2}\left(10^{26} \mathrm{~Wb}^{2}\right)$, but the observed processes whereby it is thought to be injected into the corona do not yet provide an accurate estimate of the resulting magnetic helicity budget or time evolution. The variation in magnetic helicity is important for understanding the physics of flares, coronal mass ejections, and their associated magnetic clouds. To shed light on this topic, we investigate here the changes in magnetic helicity due to electric currents in the corona for a single twisted flux tube that may model characteristic coronal structures such as active region filaments, sigmoids, or coronal loops.

Methods. For a bipolar photospheric magnetic field and several distributions of current, we extrapolated the coronal field as a nonlinear force-free field. We then computed the relative magnetic helicity, as well as the self and mutual helicities.

Results. Starting from a magnetic configuration with a moderate amount of current, the amount of magnetic helicity can increase by 2 orders of magnitude when the maximum current strength is increased by a factor of 2 . The high sensitivity of magnetic helicity to the current density can partially explain discrepancies between measured values on the photosphere, in the corona, and in magnetic clouds. Our conclusion is that the magnetic helicity strongly depends on both the strength of the current density and also on its distribution.

Conclusions. Only improved measurements of current density at the photospheric level will advance our knowledge of the magnetic helicity content in the solar atmosphere.
\end{abstract}

Key words. Sun: corona - Sun: magnetic fields - Sun: flares - Sun: coronal mass ejections (CMEs)

\section{Introduction}

Magnetic helicity is an important quantity in the physics of eruptive events occurring in the solar corona. Active region filaments and sigmoids have been observed and modelled as twisted flux bundles (Rust \& Kumar 1996; Aulanier \& Démoulin 1998; Canfield et al. 1999; Aulanier et al. 1999; Régnier et al. 2002; Gibson et al. 2002; Régnier \& Amari 2004; Török \& Kliem 2003). Magnetic helicity is also thought to play an important role in the formation of filaments (e.g., Mackay et al. 1997; Mackay \& van Ballegooijen 2005) and possibly in coronal heating (e.g., Heyvaerts \& Priest 1984; Priest 1999). The instability of coronal structures is related to the amount of helicity stored in the magnetic field and the possible transfer of twist to writhe or self helicity from mutual helicity.

Nevertheless, only proxies of the magnetic helicity have been used to estimate the twist of magnetic structures. In Leamon et al. (2004), the linear force-free parameter $\alpha$ was derived as a proxy for the twist of coronal structures observed in X-rays based on the thin flux tube approximation. The twist values of the coronal structures and of the associated magnetic clouds are inconsistent, suggesting that the propagation of coronal mass ejections (CMEs) in the corona involves a transfer between the mutual helicity of the overlying field and the self helicity of the flux rope. Based on a linear force-free assumption, Démoulin et al. (2002) have shown that the helicity of magnetic clouds is comparable to the end-to-end helicity of the twisted bundle formed in the associated active region. This suggests that the helicity is more likely to be injected through the photosphere by flux emergence or localized magnetic field motions (see also Chae 2001; Kusano et al. 2002; Welsch \& Longcope 2003; Magara \& Longcope 2003; Longcope et al. 2007). The helicity injection processes play a key role in the long-term evolution of solar magnetic field (e.g., Yeates et al. 2008). The helicity of magnetic clouds, however, is often found to be one or two orders of magnitude greater than the estimated coronal helicity. In this Letter, we model coronal structures by a single twisted flux tube with several distributions of current density and study the variations in the helicity content due to an increase in current density.

In reconstructed magnetic fields, the magnetic energy increases when the current density is increased; however, we can only inject a finite amount of current into a finite domain of computation. An upper limit of the amount of free magnetic energy is given by the Aly-Sturrock (AS) limit (Aly 1984; Sturrock 1991) stating that, for a magnetic field strength decaying fast enough at infinity in the half space above the photosphere, the magnetic energy of the open magnetic field is the least upper bound of the magnetic energy of a force-free field. Thus, the magnetic helicity that can be injected into a magnetic configuration is also bounded. The magnetic energy of the open field configuration is about twice the magnetic energy of the potential field (e.g., Amari et al. 2000). Both the potential field energy and open field energy depend of the total unsigned flux magnetic field through the surface. In a finite domain of computation as is the case for magnetic field extrapolations, the AS limit is used to check the 
validity of a magnetic configuration, therefore the extrapolated configuration is considered valid when the magnetic energy of the force-free field is lower than the magnetic energy of the open field. This condition is true when closed boundary conditions are used to derive the nonlinear force-free field.

\section{Magnetic field and current distributions}

The first step in order to derive the magnetic helicity content in the corona is to compute the 3D magnetic field in a finite volume. We assume that the coronal magnetic field is described well by a nonlinear force-free field satisfying the following equations: $\boldsymbol{\nabla} \wedge \boldsymbol{B}=\alpha \boldsymbol{B}$, where $\alpha$ is the force-free function depending on the position, and $\boldsymbol{B} \cdot \nabla \alpha=0$, which implies that $\alpha$ is a constant along a given field line. The magnetic field also has to satisfy the solenoidal condition $(\boldsymbol{\nabla} \cdot \boldsymbol{B}=\mathbf{0})$. We solve this problem in accordance with the method developed by Grad \& Rubin (1958). Following Sakurai (1981), the boundary conditions for solving this set of equations as a mathematically well-posed boundary problem are the vertical component of the magnetic field everywhere on the surface $\delta \Omega$, and the distribution of $\alpha$ in one chosen polarity on $\delta \Omega^{ \pm}$. We use the numerical scheme developed by Amari et al. (1997; 1999), which was successfully applied to solar active regions by Régnier et al. $(2002,2004,2006)$ by qualitatively comparing the computed field lines to multi-wavelength observations. It is important to note that we use closed boundary conditions on the sides of the computational box, different from the bottom boundary, which are compatible with the boundary conditions used to derive the helicity integrals (see Sect. 3).

The second step is to define the appropriate boundary conditions on the bottom boundary: the vertical magnetic field on the surface and the distribution of the force-free function $\alpha$ in one chosen polarity. As depicted in Fig. 1 left, the vertical magnetic field on the bottom boundary is a bipolar field embedded in a large field-of-view in order to minimise the influence of the boundaries on the magnetic field configurations. The vertical magnetic field component $B_{z}$ is described by a Gaussian distribution (see Fig. 1a) with a maximum field strength of $2000 \mathrm{G}$ and a full-width at half-maximum (FWHM) of about $15 \mathrm{Mm}$. The field-of-view is $150 \times 150 \mathrm{Mm}^{2}$ with a spatial resolution of $1 \mathrm{Mm}$. The peak-to-peak separation of the polarities is about $30 \mathrm{Mm}$.

The vertical current distributions are defined in the positive polarity as follows:

- Constant: $\alpha$ is a constant (see Fig. 1b) corresponding to a linear force-free field. The current distribution is then a Gaussian distribution with the same FWHM as $B_{z}$ and a maximum current density strength $J_{z 0}$.

- Divided: the distribution is defined from a Hermite polynomial of 1st order dividing the polarity into a negative and a positive part (see Fig. 1c):

$$
J_{z}(r)=2 J_{z 0} r \exp \left(-\frac{r^{2}}{\sigma^{2}}\right),
$$

where $r$ is the distance from the centre of the polarity. The current density flux through the positive polarity at the bottom boundary is then balanced. A free parameter of the current distribution is the angle $\theta$ between the polarity inversion line (PIL) and the current inversion line (CIL) in the positive polarity. We have chosen $\theta=0$ (with the negative currents towards the PIL) such that the magnetic energy is maximised.
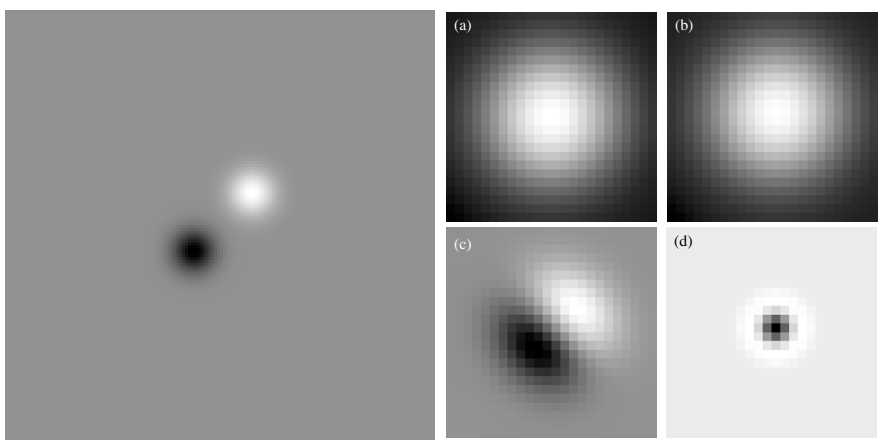

Fig. 1. Left: distribution of the vertical component of the magnetic field on the bottom boundary. The polarities are defined as Gaussian distributions with the same maximum strength in absolute value and the same FWHM. The field-of-view is $150 \times 150 \mathrm{Mm}^{2}$. The negative (positive) polarity is black (white). Right: a) vertical magnetic field component in the positive polarity; b) Gaussian distribution of the vertical current corresponding to a constant $\alpha$ field; c) divided distribution of current; d) ring distribution of current.

- Ring: the vertical current density is defined as a Hermite polynomial of 2 nd order in 1D:

$$
J_{z}(r)=2 J_{z 0}\left(r^{2}-C_{0}\right) \exp \left(-\frac{r^{2}}{\sigma^{2}}\right)
$$

where $r$ is the distance from the centre of the polarity. A typical ring distribution is plotted in Fig. 1d with negative currents in the central region surrounded by positive currents. The constant $C_{0}$ is such that the vertical current density in the positive polarity is balanced.

\section{Helicity measurements}

\subsection{Magnetic helicities}

The magnetic helicity describes the complexity of the field in terms of its topology, connectivity or braiding, and it is a measure of both the twist of field lines around the flux bundle axis and writhe of the axis itself (Berger 1999). The magnetic helicity is a conserved quantity in ideal MHD for a volume bounded by a surface on which the normal field component is fixed. However, the magnetic helicity is not conserved whilst modelling the solar corona above the photosphere since helicity may be injected from below the photosphere into the corona (e.g., Régnier \& Canfield 2006) or it may be expelled in magnetic clouds during CMEs into the interplanetary medium.

The magnetic helicity is defined as follows:

$H_{\mathrm{m}}(\boldsymbol{B})=\int_{\Omega} \boldsymbol{A} \cdot \boldsymbol{B} \mathrm{d} \Omega$

for a magnetic field $\boldsymbol{B}$ and its vector potential $\boldsymbol{A}$ in a volume $\Omega$. The vector potential is not defined uniquely but depends on a gauge. Here we use a gauge-free expression of the magnetic helicity due to Berger \& Field (1984) and called the relative magnetic helicity

$\Delta H_{\mathrm{m}}\left(\boldsymbol{B}, \boldsymbol{B}_{\mathrm{pot}}\right)=\int_{\Omega}\left(\boldsymbol{A}-\boldsymbol{A}_{\mathrm{pot}}\right) \cdot\left(\boldsymbol{B}+\boldsymbol{B}_{\mathrm{pot}}\right) \mathrm{d} \Omega$,

where $\boldsymbol{B}$ and $\boldsymbol{A}$ describe the magnetic field of the configuration, and, $\boldsymbol{B}_{\text {pot }}$ and $\boldsymbol{A}_{\text {pot }}$ describe a reference field taken to be the potential field. We use the same boundary conditions as for the universal helicity formula derived by Hornig (2006). 
Following Berger (1999), we define the self and mutual helicities as

$H_{\mathrm{self}}\left(\boldsymbol{B}_{\mathrm{cl}}\right)=\int_{\Omega} \boldsymbol{A}_{\mathrm{cl}} \cdot \boldsymbol{B}_{\mathrm{cl}} \mathrm{d} \Omega$

and

$H_{\mathrm{mut}}\left(\boldsymbol{B}_{\mathrm{pot}}, \boldsymbol{B}_{\mathrm{cl}}\right)=2 \int_{\Omega} \boldsymbol{A}_{\mathrm{pot}} \cdot \boldsymbol{B}_{\mathrm{cl}} \mathrm{d} \Omega$

when the field $\boldsymbol{B}$ can be decomposed into two fields, the reference field $\boldsymbol{B}_{\mathrm{pot}}$ and the closed field $\boldsymbol{B}_{\mathrm{cl}}$. The boundary conditions are explained in Régnier et al. (2005) and they are the same as used to compute the nonlinear force-free field in this experiment.

We note that those definitions of self and mutual helicities are different from the recent definitions given by Longcope \& Malanushenko (2008). In the solar context, the self and mutual helicities as derived by Berger (1999) have been defined in Régnier et al. (2005) from measurements based on simple configurations and observed active regions. The self helicity is a measure of the twist and writhe of flux bundles confined in the coronal volume. The mutual helicity characterises the crossing of field lines and the large-scale twist.

\subsection{Helicities vs. current}

We now compute the nonlinear force-free field in the corona for the three different distributions of current, and we study the changes in the magnetic configurations caused by an increase in the maximum vertical current strength $J_{z 0}$. In a forthcoming paper, we will extensively study the changes in the geometry of field lines, the magnetic connectivity, and the magnetic energy budget. In this Letter, we focus our study on the changes in the magnetic helicity content of the bipolar field for the three different current distributions described in Sect. 2. The maximum current strength ranges from 0 to $24 \mathrm{~mA} \mathrm{~m}^{-2}$. We first plot the total unsigned current inside $\Omega$ as a function of $J_{z 0}$ in Fig. 2 for the three current distributions. In Fig. 3, the self and mutual helicities are the blue and green curves, respectively, whilst the total relative magnetic helicity is the red curve. We also indicate the AS limit when the magnetic energy of the nonlinear force-free field is 1.7 times the magnetic energy of the potential field, corresponding to the magnetic energy computed for the open field. This upper limit gives $J_{z 0}=6.6 \mathrm{~mA} \mathrm{~m}^{-2}$ for the constant distribution, $13.6 \mathrm{~mA} \mathrm{~m}^{-2}$ for the divided distribution. For this range of $J_{z 0}$ values, there is no upper limit for the ring distribution, which indeed corresponds to a twisted flux bundle confined by return currents.

The magnetic helicities have a positive sign, except for the relative magnetic helicity of the ring distribution below $J_{z 0}=$ $5 \mathrm{~mA} \mathrm{~m}^{-2}$ (see Fig. 3c). From these computations, the mutual helicity values are most sensitive to the existence of an upper bound for the magnetic energy. For the constant and divided distributions (see Figs. 3a,b), the behaviour of the mutual helicity is strongly modified above the AS limit, whilst we get a smooth curve of mutual helicity for the ring distribution.

For all of the imposed current distributions, the magnetic helicity of the bipolar field is dominated by the self helicity, and thus the configurations are twisted flux tubes confined in a small domain of the computational box according to the definition given in Régnier et al. (2005). The helicity values vary from $10^{38}$ to $10^{42} \mathrm{G}^{2} \mathrm{~cm}^{4}$ for the constant and divided distributions, from $10^{37}$ to $10^{41} \mathrm{G}^{2} \mathrm{~cm}^{4}$ for the ring distributions. The ring distribution tends to reduce the amount of magnetic helicity stored

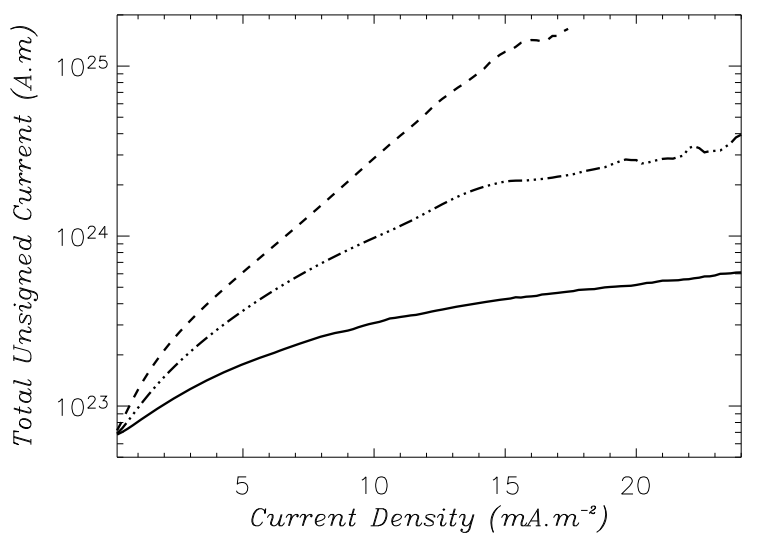

Fig. 2. Total unsigned current (A m) inside the computational volume as a function of the current density $J_{z 0}$ for a constant distribution (dashed line), a divided distribution (dot-dashed line), and a ring distribution (solid line).

in the confined twisted flux tube. The relative and self helicities show an exponential growth with increasing current density (linear trend in Fig. 3). For moderate values of the current density, these helicities vary by more than 1 order of magnitude when the current density $J_{z 0}$ is multiplied by a factor of 2 . For high values of $J_{z 0}$, a plateau exists for all helicities. This suggests that instabilities such as kink instability can develop when the maximum helicity is reached inside the twisted flux bundle. The increase in magnetic helicity as a function of the total current is reduced compared to the evolution with respect to $J_{z 0}$, but it is still significant depending on the distribution of $J_{z}$.

\section{Discussion and conclusions}

By modelling coronal magnetic structures by a bipolar field containing a single twisted flux tube, we have demonstrated that the magnetic helicity can vary by more than an order of magnitude when the current density is increased by a factor of 2 . This shows that the departure from the potential field state has important consequences on the amount of magnetic helicity that can be stored in the corona, and therefore on the helicity content expelled during CMEs.

In terms of magnetic field modelling, we have shown that the behaviour of the magnetic helicity in a nonlinear force-free field strongly depends on the chosen distribution of current. If we inject a large amount of current density within the ring distribution, the magnetic helicity values are one order of magnitude lower than for the divided distribution as the twisted flux tube remains confined to a small fraction of the coronal volume. This is caused by the existence of return currents at the edges of the twisted flux bundle, whilst for the constant and divided current distributions, the field lines can expand towards the boundaries.

In terms of observation, the vertical current density is deduced from vector magnetic field measurements in the photosphere (or in the chromosphere): $J_{z}^{\text {phot }}=\frac{1}{\mu_{0}}\left(\frac{\partial B_{y}^{\text {phot }}}{\partial x}-\frac{\partial B_{x}^{\text {phot }}}{\partial y}\right)$. The uncertainties on the vertical current density strongly depend on the noise level of the transverse field components inverted from spectropolarimetric observations. From the above conclusions, it is worth noticing that magnetic helicity values in the corona obtained from photospheric observations have to be considered with caution as a small error on the measurement of the field components can dramatically change the value of the magnetic helicity. And so to better understand the physics of twisted flux 

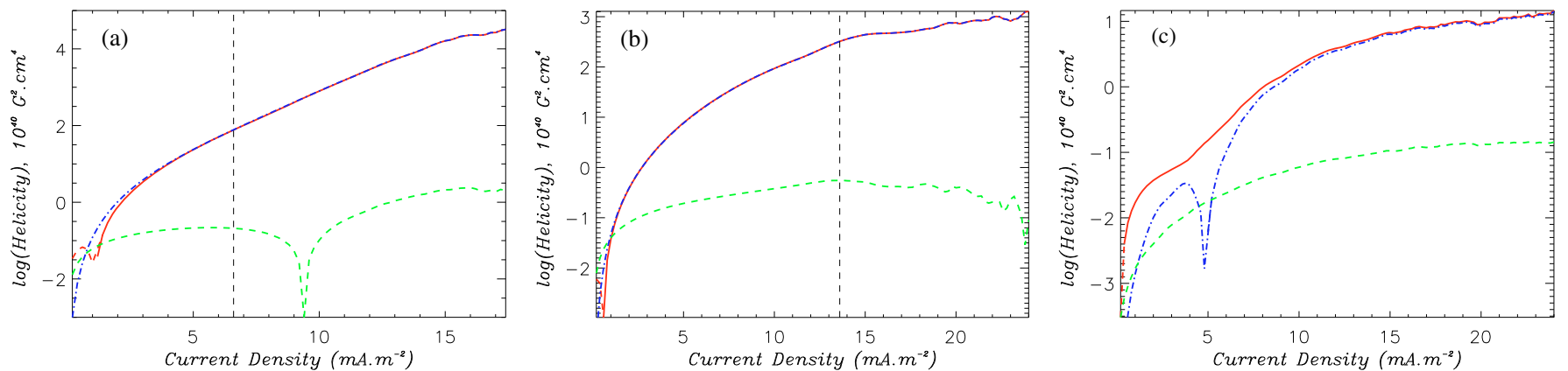

Fig. 3. Relative magnetic helicity (red solid line), self helicity (blue dot-dashed line), and mutual helicity (green dashed line) as a function of the maximum vertical current density $J_{z 0}$ from 0 to $24 \mathrm{~mA} \mathrm{~m}^{-2}$ for a) a constant distribution; b) a divided distribution; c) a ring distribution. The helicities are expressed in units of $10^{40} \mathrm{G}^{2} \mathrm{~cm}^{4}$. The straight dashed lines indicate the Aly-Sturrock limit.

bundles in the corona, it is important to improve the polarimetric resolution to increase the signal-to-noise ratio on the transverse field components and to increase the spatial resolution to resolve the current which is distributed on small scales (e.g., Parker 1996). Even if the effects are weaker, these conclusions also apply to the change in helicity as a function of the total unsigned current.

Acknowledgements. I thank E. R. Priest, D. Mackay, and G. Hornig for fruitful discussions. I thank the UK STFC for financial support (STFC RG). The computations were done using the XTRAPOL code developed by T. Amari (Ecole Polytechnique, France). I also acknowledge the financial support by the European Commission through the SOLAIRE network (MTRN-CT-2006-035484).

\section{References}

Aly, J. J. 1984, ApJ, 283, 349

Amari, T., Aly, J. J., Luciani, J. F., Boulmezaoud, T. Z., \& Mikic, Z. 1997, Sol. Phys., 174, 129

Amari, T., Boulmezaoud, T. Z., \& Mikic, Z. 1999, A\&A, 350, 1051

Amari, T., Luciani, J. F., Mikic, Z., \& Linker, J. 2000, ApJ, 529, L49

Aulanier, G., \& Démoulin, P. 1998, A\&A, 329, 1125

Aulanier, G., Démoulin, P., Mein, N., et al. 1999, A\&A, 342, 867

Berger, M. A. 1999, in Measurement Techniques in Space Plasmas Fields, ed. M. R. Brown, R. C. Canfield, \& A. A. Pevtsov, 1

Berger, M. A., \& Field, G. B. 1984, J. Fluid Mech., 147, 133

Canfield, R. C., Hudson, H. S., \& McKenzie, D. E. 1999, Geophys. Res. Lett., 26,627
Chae, J. 2001, ApJ, 560, L95

Démoulin, P., Mandrini, C. H., van Driel-Gesztelyi, L., et al. 2002, A\&A, 382, 650

Gibson, S. E., Fletcher, L., Del Zanna, G., et al. 2002, ApJ, 574, 1021

Grad, H., \& Rubin, H. 1958, in Proc. 2nd Int. Conf. on Peaceful Uses of Atomic Energy, Geneva, UN, 31, 190

Heyvaerts, J., \& Priest, E. R. 1984, A\&A, 137, 63

Hornig, G. 2006, ArXiv Astrophysics e-prints

Kusano, K., Maeshiro, T., Yokoyama, T., \& Sakurai, T. 2002, ApJ, 577, 501

Leamon, R. J., Canfield, R. C., Jones, S. L., et al. 2004, J. Geophys. Res., Space Phys., 109, 5106

Longcope, D. W., \& Malanushenko, A. 2008, ApJ, 674, 1130

Longcope, D. W., Ravindra, B., \& Barnes, G. 2007, ApJ, 668, 571

Mackay, D. H., \& van Ballegooijen, A. A. 2005, ApJ, 621, L77

Mackay, D. H., Gaizauskas, V., Rickard, G. J., \& Priest, E. R. 1997, ApJ, 486, 534

Magara, T., \& Longcope, D. W. 2003, ApJ, 586, 630

Parker, E. N. 1996, ApJ, 471, 485

Priest, E. R. 1999, in Measurement Techniques in Space Plasmas Fields, ed. M. R. Brown, R. C. Canfield, \& A. A. Pevtsov, 141

Régnier, S., \& Amari, T. 2004, A\&A, 425, 345

Régnier, S., \& Canfield, R. C. 2006, A\&A, 451, 319

Régnier, S., Amari, T., \& Kersalé, E. 2002, A\&A, 392, 1119

Régnier, S., Amari, T., \& Canfield, R. C. 2005, A\&A, 442, 345

Rust, D. M., \& Kumar, A. 1996, ApJ, 464, L199

Sakurai, T. 1981, Sol. Phys., 69, 343

Sturrock, P. A. 1991, ApJ, 380, 655

Török, T., \& Kliem, B. 2003, A\&A, 406, 1043

Welsch, B. T., \& Longcope, D. W. 2003, ApJ, 588, 620

Yeates, A. R., Mackay, D. H., \& van Ballegooijen, A. A. 2008, Sol. Phys., 247, 103 